\title{
V1062 Tau - comparison of the X-Ray (INTEGRAL) and optical (D50) data
}

\section{Martin Blazek*}

Czech Technical University in Prague, Faculty of Electrical Engineering and

Astronomical Institute of the Academy of Sciences of the Czech Republic

E-mail: martin.blazek@fel.cvut.cz

\section{Rene Hudec}

Czech Technical University in Prague, Faculty of Electrical Engineering and

Astronomical Institute of the Academy of Sciences of the Czech Republic

E-mail: rene.hudec@gmail.com

We present the multifrequency analysis of the nova-type cataclysmic variable star V1062 Tau based on INTEGRAL / IBIS X-Ray data, robotic telescope D50 optical data and other instruments.

8th INTEGRAL Workshop "The Restless Gamma-ray Universe" - Integral2010, September 27-30, 2010

Dublin Ireland

\footnotetext{
* Speaker.
} 


\section{Introduction}

V1062 Tau $(1 \mathrm{H} 0459+248)$ is classified as intermediate polar at distance larger than $500 \mathrm{pc}$ and can be found on the equatorial position (FK5)

$$
\begin{aligned}
& \alpha=05^{h} 02^{m} 27.48^{s} \\
& \delta=+24^{\circ} 45^{\prime} 23.2^{\prime \prime}
\end{aligned}
$$

and has the Galactic coordinate

$$
l=178.08^{\circ}, b=-10.31^{\circ}
$$

It was discovered in X-Ray with the HEAO 1 Scanning Modulation Collimator (A3 experiment) and the Large-Area Sky Survey (A-1 experiment). A low resolution spectrum with EXOSAT indicated that the source is a cataclysmic variable [4], a long orbital period IP. Its X-Ray pulsations have been confirmed by RXTE and ASCA data. The optical counterpart was identified as UV bright star with spectral features characteristic of a cataclysmic variable. Optical photometry shows the orbital modulation to be persistent. At shorter periods either the spin or (the newly discovered) beat period is observed. Outbursts and a possible low state are also suggested in [3]. Further X-ray observations with ASCA and RXTE confirmed the suggested classification of the system and refined the spin period [2] to

$$
P_{\text {spin }}=61.73 \pm 13 \mathrm{~min}
$$

Figure 1 (upper left panel) shows the mosaic obtained with INTEGRAL/IBIS data from April 2006, in which is clearly visible the detection of V1062 Tau in the 15-25 keV energy range (4.14 $\sigma$ significance level). Long-term observations of low and high states in multifrequency bands were therefore suggested for this cataclysmic variable.

\section{Instruments}

\subsection{INTEGRAL}

International Gamma-Ray Astrophysical Observatory [5] is ESA project providing valuable data in Gamma-Ray and X-Ray astronomy. Public data (since 2005) from IBIS instrument provided by ISDC ${ }^{1}$ (INTEGRAL Science Data Center) were processed [1] in the spectral bands $15-25 \mathrm{keV}$, $25-40 \mathrm{keV}, 40-60 \mathrm{keV}$ and 60-80 keV. The resulting light curves are shown in Figure 2 (second and third panels) together with those of other instruments. Significant evidences (upper $3 \sigma$ ) are marked with circles and error bars, while the remaining upper limits are marked with triangles. These light curves can be compared with those of optical V-band OMC instrument onboard INTEGRAL in the bottom panels of Figure 2. There is coincidence of higher activity between OMC optical data and 15-25 keV band from IBIS in February 2009.

\footnotetext{
${ }^{1} \mathrm{http}: / /$ www.isdc.unige.ch/
} 

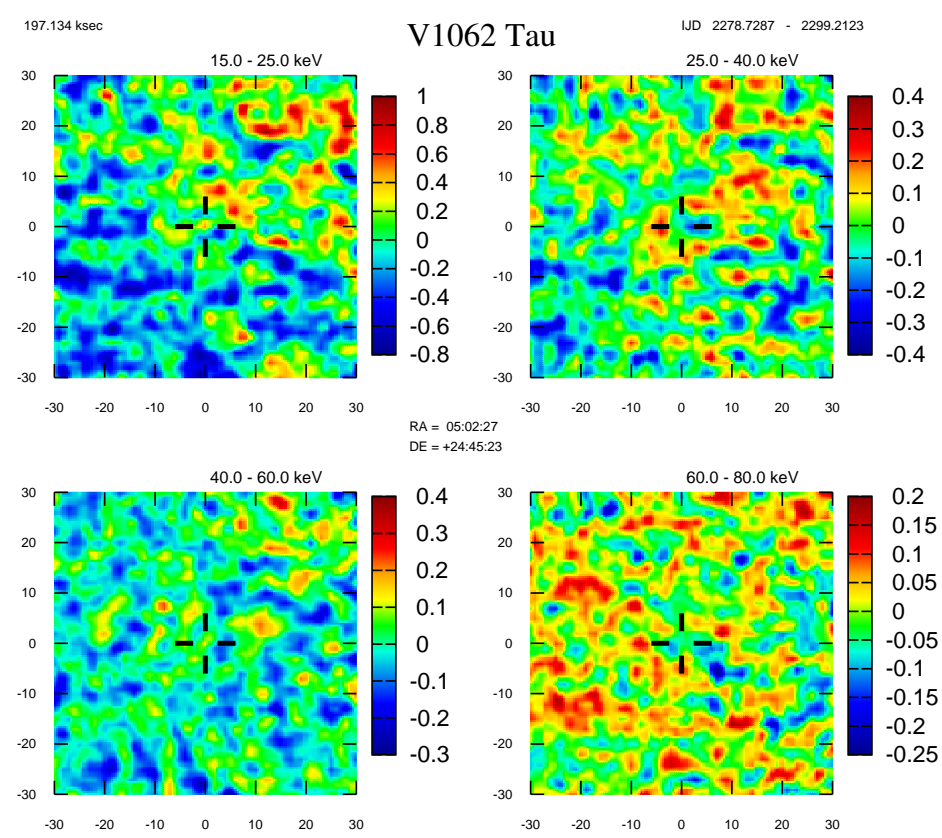

Figure 1: Mosaic of V1062 Tau from INTEGRAL/IBIS data for April 2006 in the range 15-80 keV.

\subsection{D50}

D50 is the name of autonomous robotic telescope with diameter of $0.5 \mathrm{~m}$ located in Ondrejov observatory (CZ). Long-term monitoring campaign of cataclysmic variables and blazars discovered by INTEGRAL and observable by D50 is now part of its scientific program. The optical data obtained after 2 years of follow-up observations in standard BVRI Johnson filters of V1062 Tau are shown in Figure 2. The data indicate a high state activity in November 2009, which is not confirmed by the INTEGRAL data because of the lack of public data in this period. This brightening very probably represents a low-amplitude short flare analogous to those previous reported for the system [3].

\subsection{RXTE}

Rossi X-Ray Timing Explorer was launched in 1995 and data from its ASM instrument ${ }^{2}$ for V1062 Tau are displayed in the top panel of Figure 2 (spectral range 2-10 keV). This light curve represents the one-day average of the fitted source fluxes from more individual ASM dwells.

\section{Conclusion}

Our analysis of INTEGRAL/IBIS data allowed us to detect the cataclysmic variable V1062 Tau in several observation periods with the significance confidence upper $3 \sigma$ level. By comparing those findings with the results obtained with other instruments, we provided a wider multifrequency analysis of this source. Those results confirm the importance of automated telescopes in providing

\footnotetext{
${ }^{2} \mathrm{http} / / /$ xte.mit.edu/asmlc/ASM.html
} 

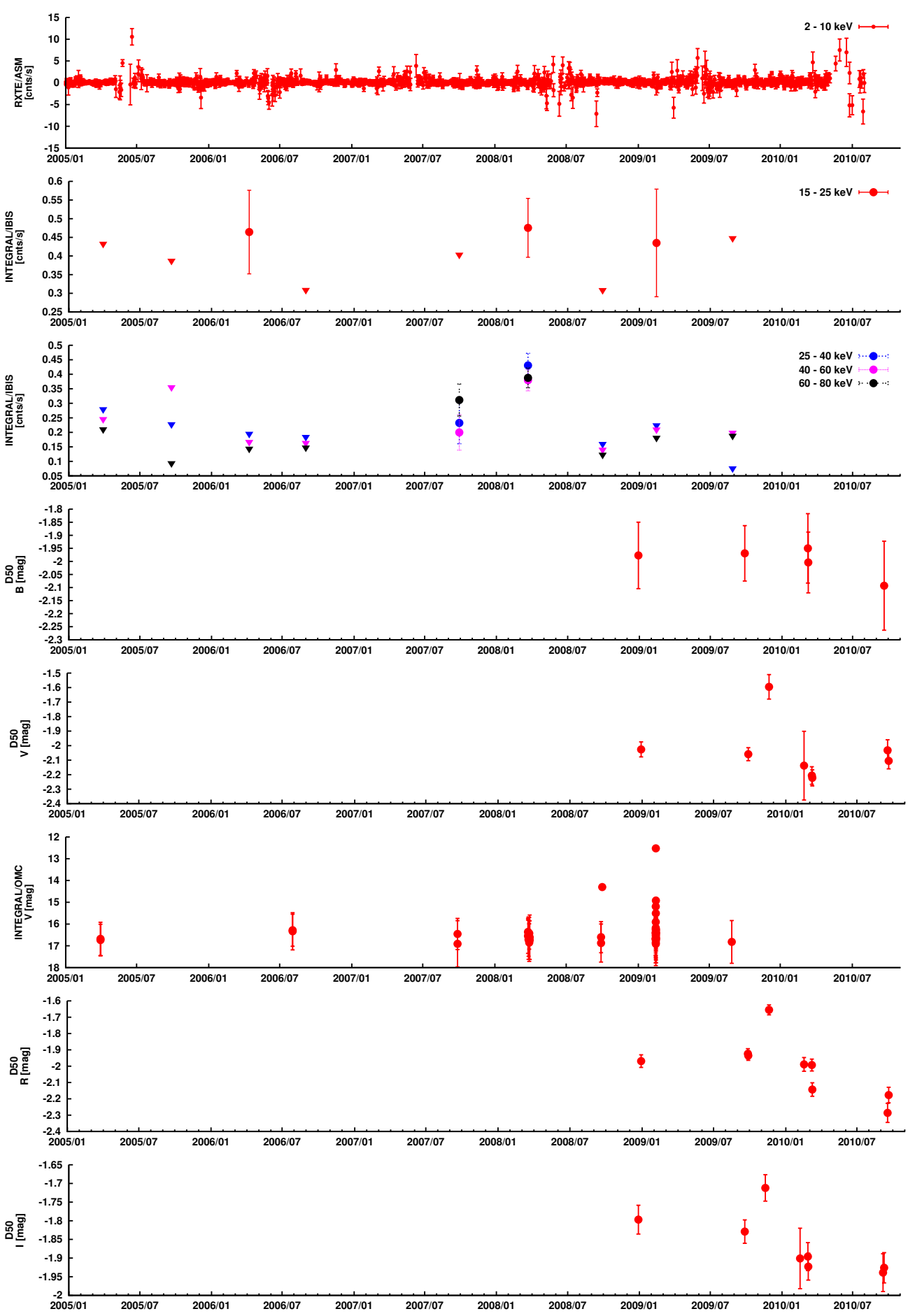

Figure 2: Comparison of the V1062 Tau light curves obtained from different instruments. Significant points are marked with round points with error bars while $3 \sigma$ upper limits are represented with small triangles. The top three panels show X-Ray data from RXTE/ASM and INTEGRAL/IBIS covering the spectral range between 2 and $80 \mathrm{keV}$. The remaining bottom panels display of optical light curves from robotic telescope D50 and INTEGRAL/OMC. 
optical monitoring data for satellites. We suggest longer monitoring campaign by ground robotic telescopes and a synergy of space telescopes for multiwavelengths follow-up observations of high states.

\section{Acknowledgments}

This study was supported by grants GA CR 205/08/1208, GA CR 102/09/0997, SGS grant 10/285/OHK3/3T/13 of CVUT FEE in Prague and ESA PECS project 98023. The International Gamma-Ray Astrophysics Laboratory (INTEGRAL) is an European Space Agency mission with instruments and science data center funded by ESA member states (especially the PI countries: Denmark, France, Germany, Italy, Spain, Switzerland), Czech Republic and Poland, and with the participation of Russia and the USA. This research has made use of the SIMBAD database, operated at CDS, Strassbourg, France. Data were provided by ISDC, D50 team (Czech Republic) and NASA/RXTE.

\section{References}

[1] Goldwurm A., The INTEGRAL/IBIS scientific data analysis, A\&A, 411, p. L223-L229 (2003)

[2] Hellier C., Beardmore A. P., Mukai K., The spin pulse of the intermediate polar V1062 Tauri, A\&A, 389, p. 904-907 (2002)

[3] Y. M. Lipkin, E. M. Leibowitz, M. Orio, Phototmetry of V1062 Tau: low states, short outbursts, and period-switching, MNRAS, 349, p. 1323-1330 (2004)

[4] Remillard R. A., Bradt H. V., Brissenden R. J. V., Buckley D. A. H., Schwartz D. A., Silber A., Stroozas B. A., and Tuohy I. R., The X-ray selected cataclysmic variables H0459 + 246 and H0857 242, ApJ, 428, p. 785-796 (1994)

[5] Winkler C. et al., The INTEGRAL mission, A\&A, 411, p. L1-L6 (2003) 